\title{
RATE OF
}

\section{HOURLY PULSATION AND RESPIRATION}

IN PHTH ISIS,

AND

I'T'S RELATIONS TO SLEEP, FOOD, SUNLIGH'T, \&c.

WITH FOUR DIAGRAMS AND TABLES.

BY

EDWARD SMITH, M.D., LL.B., L.R.C.P.,

OF THE CHEST, BROMPTON, ETC.

Received Feb. 5th.-Read March 11th, 1856.

The following is part of an extended investigation, now in progress, at the Hospital for Consumption and Diseases of the Chest, Brompton.

The subjects were three men, who had already aided in another inquiry, and three women, and were placed in two wards apart from other patients. The rate of pulsation and respiration was ascertained at the commencement of each of 144. consecutive hours, or six days and nights, in the lying posture only. No interference with their habits was permitted, except that of lying down at five minutes before and a few minutes after each hour. Their rest, at night, was 
commonly undisturbed. The meal hours were $8 \frac{1}{2}$ a.m., 5 p.m., and $8 \frac{1}{2}$ p.m., for both sexes; and $12 \frac{1}{2}$ p.m., for the women, and nearly 1 p.m., for the men. The hour of rising was usually $8 \frac{1}{2}$ a.m., and of retiring $8 \frac{1}{2}$ p.m. No complication occurred, except a slight attack of diarrhœa in No. 80, and of hæmoptysis in No. 73.

The respirations were counted in full minutes, and the pulsations in half minutes, and during the night the former were counted by the ear, so as to avoid disturbing the patients by the touch. The touch, in counting the pulsations, occasionally awoke the patients, and, on such occasions, we determined the different rates of the functions during sleep and waking.

Nos. 73, 80, and 88, were males ; and Nos. 33, 34, and 55 , were females. The following are the physical signs in each case :

No. 73 was æt. $22,4 \mathrm{ft}$. $9 \frac{5}{8}$ in. high, of sanguine temperament, and a compositor. He had been ill ten months, and was much emaciated. His vital capacity was $90 \mathrm{C}$. I. Right lung : dull universally, prolonged expiration, less vesicular murmur, cavity. Left lung : dull universally, moist râles, prolonged expiration, very little vesicular murmur, bronchial respiration.

No. 80 , æt. $20,5 \mathrm{ft} .5 \frac{1}{4}$ in. high, spare, active, an in-door servant, ill at intervals for years, aspect nearly healthy. Right lung : dull (moderately), patches of crepitant and other moist râles. Left lung : dull, flattening, very little mobility, less respiration, less vesicular murmur, two cavities.

No. 88 , æt. $45,5 \mathrm{ft} .6_{4}^{3}$ in. high, sensitive, spare, a shipjoiner, ill eighteen months, emaciated, vital capacity 76 to 100, C. I. Right lung: dull down to fifth rib, cavity to fourth rib, moist râles, no vesicular murmur, little bronchial respiration. Left lung: dull over the clavicle, prolonged expiration, less vesicular murmur.

No. 33, æt. 40, married, ill twelve months, emaciated. Right lung: dull to second rib, harsh respiration, prolonged expiration, very short inspiration, no vesicular murmur, crepitation to fourth rib, cavity. Left lung : dull 
to second rib, prolonged expiration below, no vesicular murmur above.

No. 34, æt. 22, married, dressmaker, ill twelve months, but little emaciated, very excitable. Right lung: slight crepitation and prolonged expiration down to third rib. Left lung: dull to third rib, cavity at apex, crepitation to fourth rib, prolonged expiration, less vesicular murmur.

No. 35, æt. 41, widow, needlewoman, ill twelve months, emaciated. Right lung: dulness, prolonged expiration at the apex, less vesicular murmur. Left lung : dulness (great), less mobility, depression and cavity in second space, no vesicular murmur above, and lessened below.

Thus the ages were 20,22, 22, 40,41, and 45, and all the cases were in the stage of softening and formation of a cavity, but they took food and exercise well and slept well.

Four diagrams illustrate this paper. Nos. 1 and 2 exhibit the rate of both functions at each of 144 consecutive hours, or six days and nights; whilst No. 3 shows the results for each hour, on an average of the whole period, in each case. In No. 4 the average hourly variations of respiration in each case, and in all the cases of each sex combined, and of the temperature of the wards, are recorded.

The first part treats of the pulsation, considered first absolutely, and then in relation to various disturbing causes, and the second part, of the rate of respiration, considered in a similar manner. The quantity of air inspired, and the chemical changes effected at various periods of the day, are under examination.

I am greatly indebted to Vertue Edwards, Esq., our resident surgeon, for having taken the observations from 5 to 12 p.m., inclusive. 
PART I.

P U L S A T I O N.

\section{a. The hourly Changes in Pulsation, absolutely.}

These are shown in each case in Diagrams Nos. 1, 2, and 3.

On the average the pulse was the lowest from 1 to 5 a.m., and the highest from 10 a.m. to 9 p.m. There was an elevation of 26 pulsations per minute from 5 to 10 a.m., and a corresponding but lesser subsidence, in an equal period, from 9 p.m. to 1 a.m. From 1 to 5 a.m. the pulse was under 68 ; at 10 a.m. 91 ; at 3 p.m. 94 ; and at 6 and 7 p.m. $92 \frac{1}{2}$; whilst at midnight it was 76, when it was 8 beats above the minimum of the twenty-four hours. The lowest point during the day-light was mid-day, and then it was 10 beats above the pulsation at midnight. Between the hours of lowest pulsation at night the range was only 3 pulsations, and the highest points of the day, viz., 10 a.m., 3 p.m., 6 p.m., and 9 p.m., did not differ from each other more than 5 pulsations. After 5 a.m. the pulse rose, whilst the patient was yet asleep, more when he awoke, at about 7 a.m., still more on rising, and most of all directly after breakfast. During the day, it attained its highest point in the hour following the meal, and then subsided to a much lower point before the next meal. This was due to the meals, and therefore the hours of highest elevation, as also the height of the elevation, and the depth of the succeeding depressions, depended upon the hour and frequency of meals. At about 8 p.m. or 9 p.m., according to the influence of the supper, the pulse fell whilst the patient was yet up, but more so on going to bed, and still more so when asleep, so 
that the first minimum hour was about the fourth after retiring to rest.

The cases varied greatly in the number of pulsations, but agreed as to the periodic variations now given, as shown in the following table.

TABLE No. I.

Averages for each case for the whole week, and total averages in the two sexes.

\begin{tabular}{|c|c|c|c|c|c|c|c|c|}
\hline \multirow{2}{*}{ Hour. } & \multicolumn{3}{|c|}{ Cases. } & \multirow{2}{*}{$\begin{array}{c}\text { Men's } \\
\text { Average. }\end{array}$} & \multirow{2}{*}{$\begin{array}{l}\text { Women's } \\
\text { Average. }\end{array}$} & \multicolumn{3}{|c|}{ Cases. } \\
\hline & 73. & 80. & 88. & & & 33. & 34. & 35. \\
\hline 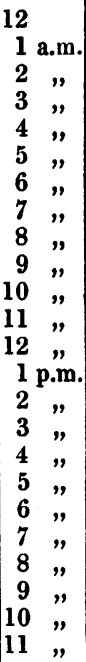 & $\begin{array}{r}91 \cdot 6 \\
88 \cdot 8 \\
86 \cdot 5 \\
80 \cdot 7 \\
82 \cdot 3 \\
80 \cdot 0 \\
82 \cdot 1 \\
84 \cdot 6 \\
83 \cdot 1 \\
95 \cdot 6 \\
104 \cdot 0 \\
103 \cdot 1 \\
97 \cdot 5 \\
98 \cdot 1 \\
98 \cdot 1 \\
103 \cdot 8 \\
100 \cdot 6 \\
97 \cdot 0 \\
102 \cdot 1 \\
103 \cdot 3 \\
101 \cdot 1 \\
95 \cdot 6 \\
93 \cdot 0 \\
89 \cdot 0\end{array}$ & $\begin{array}{l}74 \cdot 0 \\
63 \cdot 3 \\
68 \cdot 1 \\
64 \cdot 1 \\
70 \cdot 7 \\
63 \cdot 6 \\
74 \cdot 1 \\
76 \cdot 1 \\
72 \cdot 6 \\
80 \cdot 0 \\
84 \cdot 3 \\
81 \cdot 8 \\
81 \cdot 6 \\
82 \cdot 6 \\
87 \cdot 5 \\
93 \cdot 1 \\
89 \cdot 8 \\
84 \cdot 6 \\
85 \cdot 0 \\
90 \cdot 8 \\
87 \cdot 5 \\
84 \cdot 3 \\
81 \cdot 0 \\
77 \cdot 6\end{array}$ & $\begin{array}{l}73 \cdot 3 \\
70 \cdot 1 \\
69 \cdot 0 \\
66 \cdot 5 \\
64 \cdot 6 \\
65 \cdot 6 \\
65 \cdot 6 \\
74 \cdot 0 \\
75 \cdot 6 \\
93 \cdot 0 \\
94 \cdot 3 \\
86 \cdot 1 \\
84 \cdot 0 \\
85 \cdot 0 \\
89 \cdot 6 \\
90 \cdot 6 \\
85 \cdot 8 \\
87 \cdot 0 \\
89 \cdot 3 \\
87 \cdot 5 \\
84 \cdot 8 \\
87 \cdot 3 \\
83 \cdot 8 \\
77 \cdot 6\end{array}$ & $\begin{array}{l}79 \cdot 6 \\
74 \cdot 0 \\
74 \cdot 4 \\
70 \cdot 4 \\
72 \cdot 5 \\
69 \cdot 7 \\
73 \cdot 9 \\
78 \cdot 2 \\
77 \cdot 1 \\
89 \cdot 5 \\
94 \cdot 2 \\
90 \cdot 3 \\
87 \cdot 7 \\
88 \cdot 5 \\
91 \cdot 7 \\
95 \cdot 8 \\
92 \cdot 0 \\
89 \cdot 5 \\
92 \cdot 1 \\
93 \cdot 8 \\
91: 1 \\
89 \cdot 0 \\
85 \cdot 9 \\
81 \cdot 4\end{array}$ & $\begin{array}{l}73 \cdot 4 \\
68 \cdot 9 \\
68 \cdot 5 \\
67 \cdot 2 \\
67 \cdot 8 \\
66 \cdot 7 \\
69 \cdot 5 \\
72 \cdot 6 \\
78 \cdot 8 \\
83 \cdot 0 \\
88 \cdot 5 \\
87 \cdot 6 \\
85 \cdot 7 \\
89 \cdot 4 \\
92 \cdot 7 \\
91 \cdot 6 \\
90 \cdot 1 \\
90 \cdot 6 \\
92 \cdot 5 \\
91 \cdot 1 \\
90 \cdot 2 \\
88 \cdot 5 \\
85 \cdot 4 \\
81 \cdot 2\end{array}$ & $\begin{array}{l}65 \cdot 3 \\
57 \cdot 8 \\
57 \cdot 9 \\
56 \cdot 0 \\
55 \cdot 3 \\
57 \cdot 0 \\
60 \cdot 1 \\
64 \cdot 0 \\
66 \cdot 5 \\
74 \cdot 1 \\
75 \cdot 8 \\
79 \cdot 0 \\
77 \cdot 8 \\
80 \cdot 3 \\
84 \cdot 6 \\
85 \cdot 5 \\
80 \cdot 7 \\
85 \cdot 0 \\
83 \cdot 8 \\
82 \cdot 3 \\
81 \cdot 6 \\
78 \cdot 6 \\
79 \cdot 3 \\
75 \cdot 3\end{array}$ & $\begin{array}{l}77 \cdot 1 \\
76 \cdot 6 \\
76 \cdot 1 \\
76 \cdot 6 \\
75 \cdot 6 \\
72 \cdot 1 \\
74 \cdot 2 \\
77 \cdot 5 \\
82 \cdot 0 \\
81 \cdot 8 \\
94 \cdot 0 \\
90 \cdot 0 \\
87 \cdot 3 \\
91 \cdot 5 \\
94 \cdot 6 \\
91 \cdot 5 \\
92 \cdot 4 \\
92 \cdot 0 \\
94 \cdot 6 \\
94 \cdot 6 \\
95 \cdot 5 \\
89 \cdot 3 \\
83 \cdot 3 \\
79 \cdot 3\end{array}$ & $\begin{array}{l}78 \cdot 0 \\
72 \cdot 3 \\
71 \cdot 5 \\
69 \cdot 2 \\
72 \cdot 6 \\
71 \cdot 1 \\
74 \cdot 3 \\
76 \cdot 5 \\
87 \cdot 8 \\
93 \cdot 3 \\
95 \cdot 8 \\
93 \cdot 8 \\
92 \cdot 0 \\
96 \cdot 5 \\
99 \cdot 1 \\
98 \cdot 0 \\
97 \cdot 3 \\
95 \cdot 0 \\
99 \cdot 3 \\
96 \cdot 6 \\
93 \cdot 5 \\
97 \cdot 6 \\
93 \cdot 6 \\
89 \cdot 0\end{array}$ \\
\hline
\end{tabular}




\section{Range of Pulsation at various periods of the day.}

The term "range" expresses the difference between the highest and lowest observations within given periods; and the inquiry into it is as important in reference to the functions of respiration and pulsation in man as it is in meteorological inquiries.

The range of the whole 24 hours varied from 22 to 45 pulsations, both extremes being found in the same case. The average was 34 pulsations in both sexes.

The whole day was divided into four periods, viz., " night," from 1 to 5 a.m. ; " day," from 9 a.m. to 9 p.m.; " morning," from 5 a.m. to 10 a.m. ; and " evening," from 9 p.m. to 1 a.m. In the first the pulsation was at its minimum, and in the second at its maximum, whilst it rose in the morning and fell in the evening. Each period will be considered separately

1st. "Night." The average range varied from 1 to 32 pulsations in the different cases, and from 7 to 32 in the same case. It was much greater in males than in females, being 12 in the' former, and only $8 \frac{1}{3}$ in the latter. Thus the pulse varies in the quietude of the depth of the night through an average of 10 pulsations.

2d. "Day." The total average variation was 17 pulsations, and it was less in women than in men, viz., 15 in the former, and $18 \frac{1}{2}$ in the latter. The extremes were 10 and 35 in different cases; but, upon the whole, there was much more uniformity in the "day" than in the "night" pulsation.

3d. "Morning." This was very great, and on the average was not less than 27 pulsations, or 25 in the women and $28 \frac{1}{3}$ in the men; thus equaling the united ranges of the "day" and "night." The extremes were 12 and 44 , but the increase of pulsation was more uniform than those numbers indicate.

4th. "Evening." This is the only period in which the range of pulsation was greater in women than in men, 
viz., 20 in the former, and $17 \frac{1}{3}$ in the latter. The average was 19 pulsations, which was less than that of the morning, but more than those of the "day" and " night." The extremes were 8 and 32 pulsations.

Thus the four periods occupy the following order in reference to the range of pulsation, viz., "night" 10 , "day" 17, " evening" 19, and "morning" 27 pulsations. The ranges of "morning" and "evening" are nearly twice as great as those of the "day" and "night." The extremes of pulsations were the greatest in the "night." Thus the causes which influence pulsation must be more active in the "morning" and "evening" than in the developed "day" and "night." In women these influences produce a more rapid evening subsidence of the pulse, and a lower pulsation through the night than in men.

\section{The Hours and Amount of Minimum and Maximum Pulsation.}

Minimum in the night.-The total average was 65 pulsations, and was less in women than in men. The extremes in all the cases were 52 and 81 , but there was great constancy in each case separately, the variations being to the extent of 5 pulsations only. There is no relation between the minimum pulsation and extent of disease. The hour of lowest pulsation was most frequently 5 a.m., and then 6 and 4. a.m.

Minimum in the day.-This, on the average, was 84, and was nearly the same in both sexes. It was 20 pulsations above the night minimum. The extremes were considerable; viz., 65 and 100, and in each case it was greater than in the night minimum. The lowest day and the lowest night minimum were not always met with in the same case. The proportionate increase of the day over the night minimum was greatest in women; but this increase was not uniform. Thus taking the night minimum as the basis, the day increase varied as follows: Men over $\frac{1}{4}$, about $\frac{1}{4}$, and over $\frac{1}{3}$; women $\frac{1}{5}, \frac{2}{5}$, and $\frac{2}{5}$; and the total average increase 
in each case was $25,22,21 \cdot 8,18 \frac{1}{2}, 14 \frac{1}{2}$, and 14 pulsations in their order. The hour of minimum day pulsation was midday in one half of the whole observations, and then 11 a.m. and 1 p.m. each in one sixth of the observations.

Maximum pulsation in the working day.-It has already been stated that there were commonly four maxima, which, as they depend upon food, may be designated as breakfast, dinner, tea, and supper maxima.

1st. Breakfast maximum.-The average pulsation immediately after breakfast was 95 , and was the lowest in females. The extremes were 76 and 113 ; and so great a variation as 20 pulsations has been noted in the same case in different days. The hour varied from 9 to 12 a.m., but was 10 in more than a majority of instances.

2d. Dinner maximum.-This was precisely the same in both sexes, and as the average pulsation was lower in females, the maximum is proportionately higher in that sex. The hour varied from 1 to 5 p.m., and was 3 p.m. in nearly one half, and 2 p.m. in nearly one third of the observations.

3d. Tea maximum.-The total average was 96 pulsations, or a medium between that of breakfast and dinner. It was lower in women than in men. The extremes were 81 and 108 , and were somewhat less in each case than in the two former maxima. This maximum was not invariably present. The hour was from 5 to 9 p.m., but 7 p.m. was the maximum hour in one half of the observations, and then it was 6 p.m.

4th. Supper maximum.-This was not only the least of the maxima, but was the least constant, since it was found in only one half of the observations. It was less in females, as were also the breakfast and tea maxima. The extremes were 80 and 100, but the variation was inconsiderable in each case. The hour varied from 9 to 11 p.m., but was commonly 9 p.m.

Thus the four maxima were, on the average, 95, 97, 96, and 92 , in their order, showing a difference of only 5 pulsations in the average maxima of 13 hours. The dinner maximum was the highest on the average, but not in every instance: 
It was commonly so in the females, but each case varied, as we know each person to vary in the relative enjoyment of meals.

In reference to the foregoing part of the paper, we may sum up the sexual differences in the following sentences:The women had lower pulsations, with less range and extremes at night; also more limited day range, with greater proportionate increase from the night. They had a little less increase in the morning ascent, and greater decrease in the evening descent. In them also the supper maximum was less frequent.

The following comprehensive table exhibits all the information obtained in reference to maximum and minimum pulsation and range of pulsation in each of the cases, and on every day of the inquiry. 


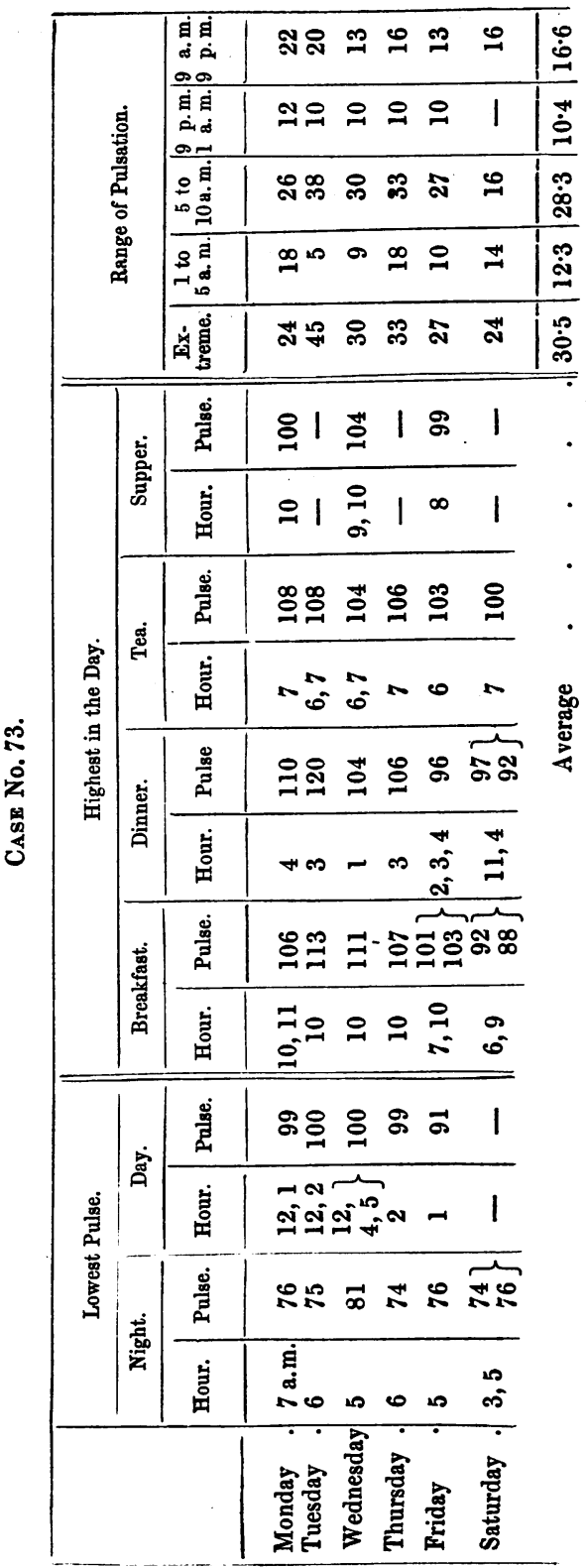




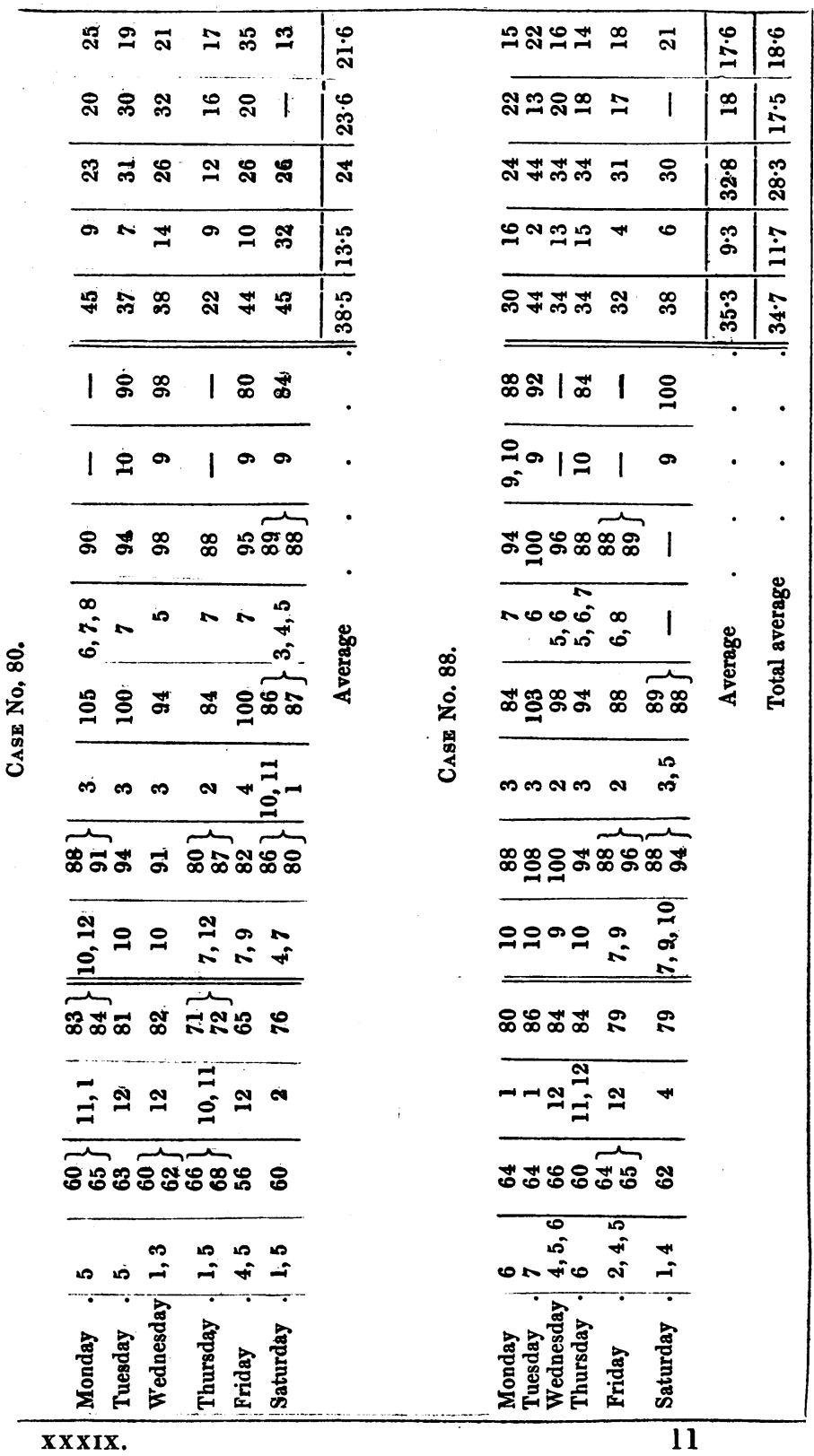




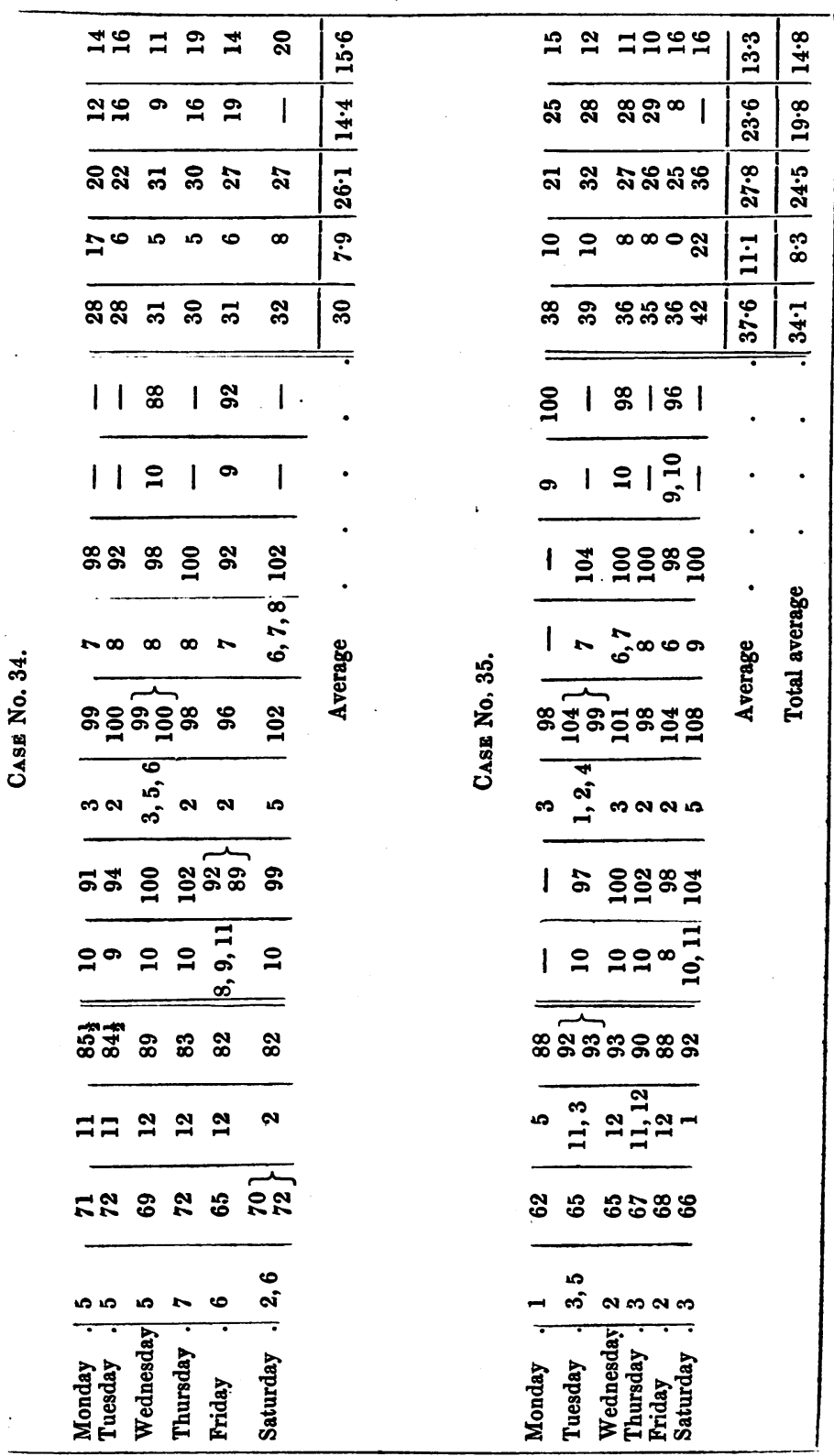




\section{b. Disturbing Influences.}

These are slcep, food, sunlight, exertion and excitement, temperature and humidity; and of these the three former had the greatest, most constant, and continued power in these investigations.

\section{Sleep.}

This is represented in diagrams Nos. 1 and 2.

It causes a depression of the pulse, whether by night or day ; but that depression is vastly greater which accompanies night sleep. Hence sleep is not necessarily associated with the lowest state of pulse, but it is probable that the soundest sleep is at night, when the pulse is low, and that sound sleep cannot occur with the elevated pulse of day. This is probably dependent upon the presence and absence of sunlight, as will be proved presently.

Waking out of sleep by night or day causes an immediate elevation of the pulse, and a permanent one so long as wakefulness continues.

Sleep at night occurs after the pulse has began to fall and whilst it is yet falling; when it is at its minimum ; and, lastly, whilst it rises to constitute the morning elevation. As the morning rise is not due to sleep, so it is probable the evening fall is partly due to other influences; or since the minimum pulsation does not occur until the second, third, or fourth hour of sleep, the effect of sleep must be accumulative. But there is no accumulation in a sccond or third hour of sleep during the day, and therefore probably none during the night. Hence it is inferred that there are other causes than sleep producing the night fall of pulsation, and, consequently, that the whole night fall is not a measure of the influence of sleep. Neither, perhaps, is the day fall from day sleep a measure of the full effects of sleep under possibly more favorable circumstances as the natural ones of night and darkness. Hence, again, the difficulty of deter- 
mining the exact influence of sleep. When sleep occurs with a falling pulse, but before the minimum pulsation has occurred at night, it causes immediately a much greater fall -one quite disproportionate to the fall before sleep; but at the middle of the night, or in the early morning, when the minimum has occurred, no such disproportionate influence is exerted if the person fall asleep from waking. Sleep, therefore, helps to drag the pulse down in the early night, to keep it down, and to prevent the morning rise.

Thus it appears to be the most powerful soon after retiring to rest; but since sleep lowers pulsation, and is believed to be the most profound at night when the pulse is much lower than during the day, it is probable that the lower the pulse during sleep, the more profound is the sleep. This lowest pulsation does not correspond with either the period of noisy or of quiet sleep, for in some cases the sleep will be as noisy or as quiet (as the case may be) in the morning at 7 o'clock, with a rising and increased pulse, as it was at 1 to $3 \mathrm{a} . \mathrm{m}$. with the minimum pulsation. I believe both the latter indications to be fallacious, but if they are valuable they would lead me to believe that in the cases under examination the relative soundness of sleep was not that of relative depth of pulsation. The depth of pulsation, and therefore of sleep also, would depend upon the hour and the nature of the last meal.

As the women had an earlier and a deeper subsidence of the pulse than the men; it may be inferred that they had sounder sleep, and at an earlier period of the night.

Measure of sleep.-The following is the measure as observed during the day in thirteen instances:-Fell $0,4,8$, $8,11,6,10,10,9,7=7 \cdot 3$ medium ; but on two occasions it rose 3 and 2 in the second hour; and once it rose 4 in the first hour, and fell 9 in the second hour. This rise, if deducted from the fall, would leave an average of 5 pulsations fall from sleep. This is sleeping under difficulties, but even then $\mathrm{I}$ am of opinion that the common subsidence of the pulse would be from 8 to 10 pulsations.

In the night the effect of sleep is doubtless greater. In 
one instance, at 4 a.m., the pulse fell from waking to sleep 18 pulsations ; but, as above stated, it varied with the period of the night. There were also numerous exceptions to the rule that the pulse falls with sleep and rises on waking; but unless we could measure the co-ordinate influences, we cannot determine the effect of sleep alone in the night. I believe it may be reasonably estimated at 10 pulsations, with the understanding that it is greater before the minimum pulsation, and less at and after the minimum. The effect of the first hour's sleep is no exact measure. It was very various, as follows :- $12,6,8,6,4,6,14,10,16,6,12$, $2,4,6,3,10,13,20,4,8,12,8,12,8,4,7,10,18,8$, and 4 ; and in a few instances there was no fall. The effect of waking was commonly to raise the pulse 8 to 10 pulsations.

This interesting inquiry may be pursued to any extent by the aid of Diagrams No. 1 and 2, due allowance being made for occasional errors in determining which was sleep and which was not sleep in the quiet repose of the night, and for coughing, involuntary attempts to repress the cough, and for mental emotions, all of which tend to raise the pulse.

\section{Effect of Food.}

The food and degree of its enjoyment are important to this inquiry, and were as follows:

Breakfast.-No. 88, egg, bread, butter, and tea; No. 80, the same, but coffee instead of tea, and bacon on June 11th; Nos. 73 and 34 as No. 80 ; No. 35, the same, without egg.

Dinner.-All took meat, bread, and rice or potatoes; Nos. 88 and 34 had porter; and Nos. 35, 73, and 80 had ale. No. 80 had only boiled rice on June 14th.

Tea.-All had bread, butter, and tea, except No. 34, who had milk. No. 73 had always an egg; and No. 80 had egg on June 13, 14, 15, and 16.

Supper.-No. 88 had cold meat, bread, and porter; No. 80 , meat, bread, and water or milk; No. 73 had milk; and Nos. 34 and 35 rice and milk.

All ate heartily and enjoyed the food, except No. 88 , 
who took a light tea on June 11, 12, 13, and 14, and had only a moderate dinner on June 16 . No. 80 had a bad dinner on June 14, and a moderate one only on the 16th. No. 73 had a small breakfast and dinner on June 16. No. 34 had two ounces of wine at about 11 a.m. daily.

The effect of food is almost uniformly to cause a temporary elevation of pulsation, so that the pulse is more frequent soon after a meal, and subsides before the forthcoming meal. Thus there are commonly as many elevations and depressions of the pulse as there are meals. The meal hours were $8 \frac{1}{2}$ a.m., $12 \frac{1}{2}$ p.m. (123 for the men), and 5 p. m., and a fourth at $8 \frac{1}{2}$ p. m., not necessarily provided by the hospital, but yet, in these investigations, always obtained. The exceptions were as follows: 1st, on the first day the men took breakfast after 9 a. m.; 2 d, Nos. 73 and 80 took dinner after 1 p.m. on the first three days, and No. 88 on the first two days; $3 \mathrm{~d}$, on the fifth day the dinner was deferred three hours. These caused a difference in the hour of elevation, for the elevations depend chiefly upon the hour and number of meals. With the above exceptions the examinations were made almost immediately after the conclusion of the meal.

The following table contains the amount of the elevations due to each meal, and also the hour of highest pulsation after the meal in each case, and on each of the six days' examination. 


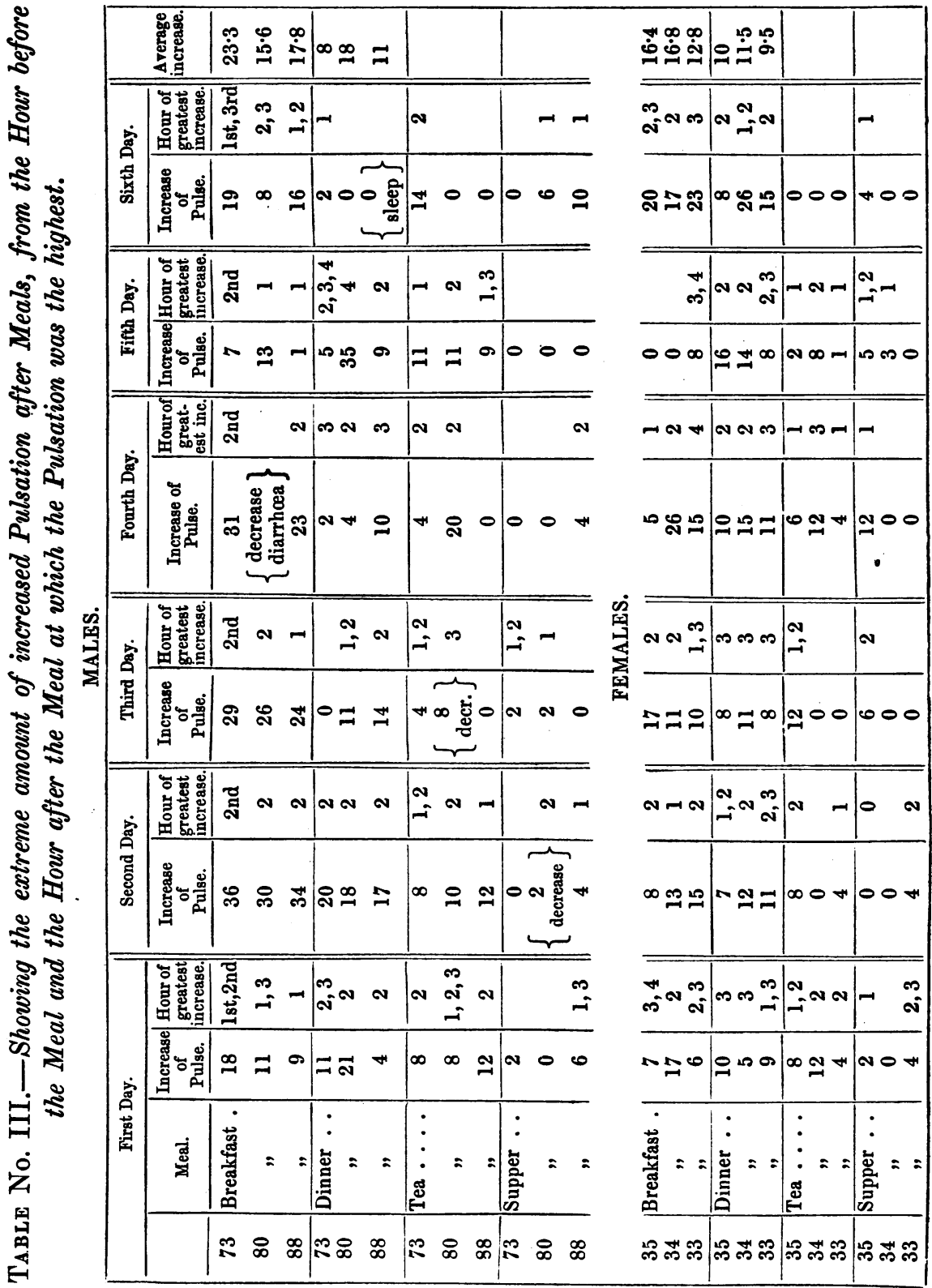




\section{Hour of Highest Elevation after a Meal.}

As the examination was not made at the end of an hour after the meal, but within half or three quarters of an hour after the commencement of the meal, it must be understood that the term means "during an hour." The maximum hour varied from the first to the fourth, but the latter was too infrequent to need observation. The second hour was as frequent as both the first and third hours combined; but there was almost always an increase during the first hour, and so frequently that that hour was second only to the second hour. Thus the order of frequency of the maximum hours were the second, first, third, and fourth. In many instances the highest pulsation occupied two or even more hours. The first was most frequently the maximum hour after tea, supper, and breakfast, in their order; whilst after dinner it occurred in only four out of thirty-seven observations. The first hour was much more frequent after breakfast, and also (but in a less degree) after supper in men; and the third hour after breakfast and dinner in women.

\section{Amount of the highest hourly Pulsation.}

It is difficult to isolate the effect of the meals from that of exercise and temperature; but with the exception of the breakfast, we shall not go far wrong in computing the elevation from the returns of the preceding hour. This rule would, however, be wrong for the breakfast, since the elevation is often then so great as 36 pulsations in the men, and 25 in the women; and there are other elements of error then operating, as those of awaking from sleep and of rising.

1. Breakfast.-I endeavoured to isolate this influence on the fifth and sixth day, by directing all the patients to rise at 6 a.m. instead of 8 a.m., and to take a walk out in the intervals of the examinations. The three men and two of the women did so on the fifth, and on the sixth morning 
two men and one woman also. On the fifth morning the highest increase from the hour before breakfast was 13, 7, and 1 pulsations in the three men, and 0 and 8 in two women. On the sixth day, it was 16 and 8 in the men. Thus the average pulsation due to breakfast is probably from 8 to 10 beats per minute; but the average increase observed, during the six days, from the hour preceding the breakfast, was $23 \cdot 3,15 \cdot 6,17 \cdot 8$ in the men, and $11 \cdot 4,16 \cdot 8$, and 12.8 in the women, or a total average of over 16 pulsations per minute.

2. Dinner. - This varied from nothing to 35 pulsations. On the average it was-men, $8,18,11=12.3$ medium; women, $10,11_{\frac{1}{2}}, 9 \frac{1}{2} \doteq 10$ medium, or a total average of 11 pulsations. Thus it was less in women, and in them also the variations were less.

3. Tea.-The average for the first few days was-men, $7,11,7=8.3$ medium; women, $7,6,3=5.3$ medium; and the total average was about 7 pulsations. The variations were less than at either of the preceding meals; viz., 1 to 20 pulsations, and they were nearly equal in the two sexes.

4. Supper.-In eleven instances in the women, and nine in the men it was null. The average increase was-men, $\frac{2}{3}$, less than $2,4=2 \frac{1}{3}$ medium ; women, $5, \frac{1}{2}, 1 \frac{1}{3}=2 \frac{1}{2}$ medium. The variations were 0 to 12 pulsations. The sexes were about equally wanting in uniformity.

Thus the average increase at each of the meals wasbreakfast 8 to 10 (estimated), dinner 11, tea 7, supper $2 \frac{1}{2}$ pulsations.

The proportion to the whole number of meals in which no increase was observed was as follows : breakfast-men 0 , women $\frac{2}{36}$; dinner-men $\frac{1}{30}$, women 0 ; tea-men $\frac{2}{30}$, women $\frac{3}{10}$; supper-men $\frac{9}{36}$, women $\frac{11}{36}$.

In the women the increase was commonly less, but more constant, than in the men, as was also the longer period before the maximum was attained after breakfast and dinner.

In this part of the investigations two other inquiries were made ; viz., the effect of early rtsing and of fasting. 


\section{The effect of Early Rising.}

This is shown in the returns of the fifth and sixth day, in cases 80 and 88 ; and on the fifth day in cases 73, 33, and 34 , all of whom then rose directly after 6 a.m, and walked round the hospital quickly between 6 and 7 a.m., and again between 7 and 8 a.m. Case 35 also rose at 7 a.m. on the fifth day, and walked out between 7 and 8 a.m. The constant effect was to produce an unusual increase of pulsation at the breakfast hour, and more particularly at the first hour after rising. In the men, the increase was confined to the first hour, and was, on the fifth day, 18, 18, 12 ; and on the sixth day, 24 and 18, or an average increase of 18 pulsations per minute. In the second hour no further increase took place; but, on the contrary, a decrease on the fifth day of 5, 5, and 1 , and the sixth day of 10 and 2 , or an average of $4 \cdot 6$ pulsations. The increase was less on the fifth than on the sixth day, because then the patients were awake at the 6 a.m. examination, and the pulse had consequently risen much. In the women, the increase on the fifth day was-first hour, 23 and 33, second hour, 4 and 2 pulsations. This increase was much greater than that of the men ; but it may be explained by stating that they had literally interpreted my directions, "to take a run round the hospital." Case 35, who rose at 7 instead of 8 a.m., and walked round the hospital, had an increase of 5 pulsations.

But the numbers now given are not entirely due to the early rising, for the pulse would have risen somewhat at that period of the morning had they remained in bed.

The excess of pulsation over the average at these hours is that due to the early rising, and was, on the total average, 10 pulsations per minute. This large and unusual amount of increase from the early rising was followed by an unusual decrease of pulsation, and hence it is desirable to know the effect of early rising on the pulsation on the first and second halves of the day, and also on the whole day. The effect 
during the morning was to increase the pulsation by an average of about 4 pulsations per minute. The increase was more than twice as great in men as in women; viz., 5,6 , men, and 2, 4, women; and consequently in the latter there was greater reaction. The pulsation in the latter half of the day showed a decrease to the extent of 4,1 , in the men, and 2,3, in the women. The effect upon the total pulsation of the whole day was a slight diminution in the rate; and, upon the whole, the effect of occasional early rising was to cause an earlier and a deeper subsidence of the pulse at night, and consequently earlier and deeper sleep.

\section{Relation of hourly temperature and humidity to pulsation.}

The returns are restricted to those obtained within the two wards of the hospital in which the males and females severally lived. That of the men's ward only was taken with both the dry and wet bulb thermometers, so that that alone will enable us to determine the degree of humidity of the air.

The progress of pulsation and temperature is shown in the following average table for each day.

TAble No. IV.

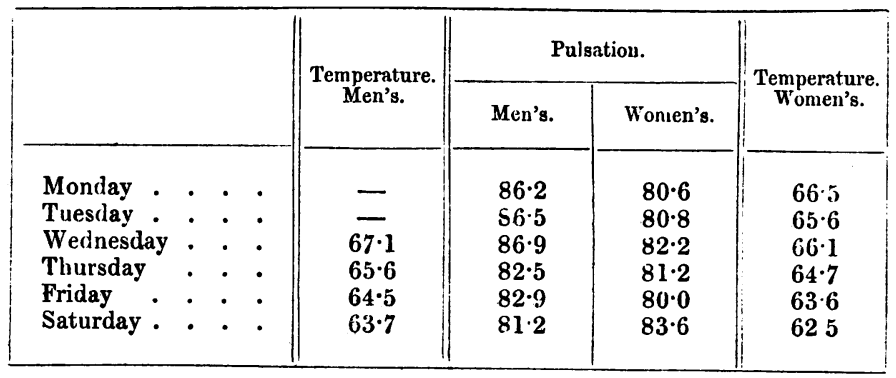

Thus the temperature declined on each day, except the third, in the women's ward ; and the pulsations of the men declined also. 
The extreme daily range in the hourly averages of the six days was only $1 \cdot 3^{\circ}$ in the women's, and $2 \cdot 2^{\circ}$ in the men's ward. The maximum in the women's ward was attained at 4 , 5 , or 6 p.m., and thenceforward the temperature declined through the night to 6 and 7 a.m. From 9 a.m. to the maximum, the increase was only $1^{\circ}$, and the temperature at midday was only $2^{\circ}$ above the minimum. In the men's ward the maximum was about midnight, and the minimum at 10 or 11 a.m. Thus the lines of temperature and pulsation so far agree, that they both decrease through the night, and increase through the day, but the maximum and minimum hours differ. Moreover, at night, the pulse falls whilst the temperature is at its highest; in the morning, it rises whilst the temperature falls to its lowest; and through the day, from 9 a.m. to 9 p.m., maintains a somewhat even relation, whilst the temperature attains its minimum, increases through the day, and nearly attains its maximum at night.

The temperature varied from hour to hour, as did the pulsation; but the line of temperature in each of the six days, when compared with the pulsation of those days, offers no instance in which the pulse appeared to be directly under the influence of hourly changes of temperature. The average hourly changes are shown in Diagram No. 4.

\section{Humidity .}

The interest of this inquiry is its relation to the warming of hospitals, for I have elsewhere proved that with elevation of seasonal temperature there is commonly increased dryness, and with both these conditions are increased pulsation and lessened rate of respiration-two conditions not tending to health.

The difference between the dry and wet bulb thermometers in the external air on the six days was $13 \cdot 1^{\circ}, 9 \cdot 9^{\circ}, 2 \cdot 9^{\circ}, 4 \cdot 2^{\circ}$, $4 \cdot 6^{\circ}$, and $2 \cdot 7^{\circ}$, showing a very great variation in one week; whilst the mean difference in the hospital was so uniform on the four last days as $6 \cdot 2^{\circ}, 6 \cdot 4^{\circ}, 6 \cdot 2^{\circ}$, and $6 \cdot 6^{\circ}$. The 
average difference on these four days between the dryness of the external and internal air was, external $3.6^{\circ}$; internal, $6.5^{\circ}$; and, consequently, on these days the internal air was twice as dry as the external ; rain fell externally on these days, and thus the humidity would be greater.

The following table gives the average hourly returns for four days of the difference between the wet and dry bulb temperature :

TAble No. V.

Hourly average difference between the wet and dry bulb Thermometers.

\begin{tabular}{|c|c|c|c|c|c|c|c|c|c|c|c|c|c|c|c|c|c|c|c|c|c|c|}
\hline \multirow[b]{2}{*}{ Hour } & \multicolumn{11}{|c|}{ a.m. } & \multicolumn{11}{|c|}{ p.m. } \\
\hline & 12 & 1 & 2 & 3 & 4 & 5 & 6 & 7 & 8 & 9 & 1011 & 18 & 1 & 2 & 3 & 4 & 5 & 6 & 7 & 8 & \begin{tabular}{l|l}
9 & 1 \\
\end{tabular} & 1011 \\
\hline Degrees & & & & & & & & & & & \begin{tabular}{l|l}
6.3 & 5.9
\end{tabular} & & & & & & & & & $6 \cdot 6$ & & $\cdot 66.4$ \\
\hline
\end{tabular}

The uniformity in the amount of humidity, as indicated by the difference between the dry and wet bulb temperatures, is very remarkable. There was a little average increase at 11 a.m. and 5 p.m., with a slight decrease at 3 p.m. Thus tolerable uniformity was attained; but the dryness was too great as compared with the external air, but equal to that for the whole of the same quarter of the year.

\section{Influence of Exercise and Excitement.}

The returns of the morning of early rising prove clearly that the pulse may be increased at an unwonted hour by exercise, but from the fact that, at 10 a.m., the pulse may attain to the same height without the patient having risen that it would have attained had he taken exercise during each of several preceding hours it is evident that the maximum pulsation of the day was uninfluenced by it. The effect of violent exercise is great but temporary, and if the 
lying posture be maintained for five or ten minutes after ordinary exercise no trace of its effect remains. Ordinary and quiet exercise had but very little influence over hourly pulsation.

\section{Effect of Sunlight.}

It has been proved that between the pulsation of the night and the day there is a difference of 25 to 40 pulsations and upwards per minute, and we have proved that sleep alone does not reduce the pulse to its great depth at night, nor food alone raise it to its great height during the day. Moreover hourly temperature has no influence that can be defined, and moderate exercise is powerless, and hence we seek for other powerful causes for this great difference. The day has light and the night the absence of it, and it will be seen that the highest pulsations occurred (in June) at the period of the day when the daylight was most powerful, that both began to increase together in the morning and to decline together in the evening. Thus sunlight is a probable cause; but I have not been able to prove it absolutely by inducing a consumptive patient to remain in absolute darkness through the day, and in the winter season the test would be insufficient. This influence may be estimated in three modes: 1st, by subtracting from the difference between the highest pulsation of the day and the lowest of the night the influence of food and of profound sleep; 2d, by taking, in like manner, the difference between the lowest pulsation of the night and the highest after the breakfast and deducting the influence of breakfast and of light sleep ; and, $3 \mathrm{~d}$, by taking the difference between the pulsation at $4 \mathrm{a}$ a.m. when it was light and mid-day, allowing a little for light sleep, and for the remaining influence of breakfast. Either would represent the influence if we could estimate each of the quantities concerned in the calculation with accuracy.

We will now investigate this subject in the three directions just indicated, and allow, according to the preceding 
inquiries, 10 pulsations for profound sleep, 10 for breakfast, 11 for dinner, and 5 each for light sleep, and for the remains of the breakfast influence at mid-day.

The average difference between the highest and lowst pulsations were $24,29,28 \cdot 7,30 \cdot 2,22 \cdot 5$, and $30=$ medium, $27 \cdot 4$. The average difference between the lowest at night and the highest after breakfast was, 24, 20.2, 30.3, 24.3, $22 \cdot 1,26 \cdot 6=24 \cdot 5$ medium. The average difference between the pulsation at 4 a.m. and mid-day was $15 \cdot 2,9 \cdot 9,20 \cdot 6$, $22 \cdot 5,11 \cdot 7$, and $19 \cdot 4=16.5$ medium. The calculations will, then, finally be-

$$
\left.\begin{array}{l}
27 \cdot 4 \text {, less Dinner } 11 \text { and Sleep } 10=6 \cdot 4 \\
16 \cdot 5 \text {, less } \frac{1}{2} \text { Breakfast } 5, \frac{1}{2} \text { Sleep } 5=6 \cdot 4 \\
24 \cdot 5 \text {, less } \frac{1}{2} \text { Sleep } 5, \text { Breakfast } 10=9 \cdot 4
\end{array}\right\} \begin{aligned}
& \text { Medium } 7 \cdot 4 \\
& \text { pulsations. }
\end{aligned}
$$

It thus appears that in consumptive patients in the month of June the sunlight exerts an influence amounting to from 6 to 10 pulsations average upon the pulsation of the day; and consequently its absence permits a corresponding reduction in the pulsation of the night. The effect in individual cases is oftentimes much greater.

\section{PART II.}

R E S P I R A T I N.

\section{Hourly Changes in the rate op Regpiration in Phthisis.}

These have been determined by the investigation already described; and also by a supplementary one made upon 29 patients on the night of November 14-15, by the kind help of Mr. V. Edwards and Mr. Vise, our talented and esteemed Resident Surgeon and Clinical Assistant respectively, 
(See diagrams, Nos. 1, 2, and 4.) In estimating the value of a relative change in the rate of respiration and pulsation the normal difference in the rate of the two functions must be remembered, and hence, that an increase of one respiration may be proportionately as great as of four pulsations.

The total average rate for both sexes exhibits a daily variation of 8 respirations with average extremes of $21 \frac{1}{2}$ and $29 \frac{1}{2}$, and absolute extremes of 13 and 49 respirations per minute. The minimum was at 8 a.m., and the maximum at midnight.

The hourly average changes are shown in the next table :

Table No. VI.

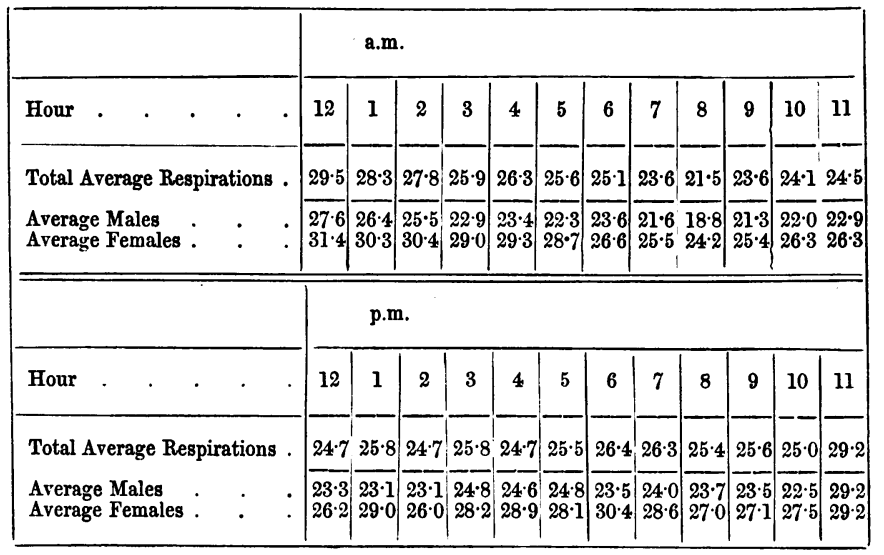

Thus, at 11 p.m. there was a sudden increase of 4.2 respirations or one sixth of the total number at 10 p.m., and then a slight increase to the maximum at midnight. Thence it declined, but remained very high at 1 and 2 a.m., and had a slight increase at 4 a.m. ; whilst at 7 a.m. and 8 a.m. the decrease was great and sudden. Thus the hour of sudden increase corresponds with that of the commencement of sleep, and of sudden decrease with that of permanent waking. At 9 a.m. it had increased 2 respirations 
from the minimum (being then the same as it was at 7 a.m.), and thence increased progressively (with a disproportionate increase at 1 p.m.) through 3 respirations to 6 p.m., and finally decreased until 10 p.m., when the sudden increase from sleep occurred.

Thus the decreasing hours were 7 to 10 p.m. and 3 to 8 a.m. (minimum), whilst the increasing were 9 a.m. to 6 p.m. and 11 p.m. to about 2 a.m. (maximum). The hours of sudden decrease were 3 a.m. and 7 and 8 a.m., and of sudden increase 11 p.m., 9 a.m., and 1 p.m.

Thus as a general expression it may be stated that the respiration increases suddenly and greatly in the early night, decreases thence to the period of rising, increases gently and progressively through the working day until after the tea hour (5 p.m.), and finally falls to and after going to bed.

The rate is greater during the night than the day. Thus in intervals of eight hours, as 11 p.m. to 6 a.m. (night) and 3 p.m. to 10 p.m. (day), and also 9 a.m. to 4 p.m. (day), the average is $27 \cdot 2$ (night), 26 and $24 \frac{1}{2}$ (day). There are exceptions to this statement; but when those are included, the rule is as just laid down.

The difference in the average returns from the two sexes was but slight. Thus, in females, there was a greater rate, a less range (7/2), and, consequently, more uniformity of respiration; a higher maximum $(31 \cdot 4)$ and minimum (24:2) rate, with the maximum one hour earlier (viz., midnight instead of 1 a.m.); and a less increase at 11 p.m. There was also a less proportionate increase after breakfast, with a greater increase at 1 and 6 p.m., and, consequently, a more tardy fall in the evening.

\section{Influence of Sleep.}

This is in general very great and decided, and tends to incfease the rate of respiration. It may be estimated in two ways-1st, by contrasting the rate in successive hours, when the patients are alternately awake and asleep; and 
2d, by ascertaining the effect of sudden waking out of sleep.

The former is liable to the following fallacies: 1st. If the full effect is not immediate, it will be influenced by the state of waking or sleeping in the intervals of the examination, and of this state we have no knowledge. 2d. The effect of sleep is in relation to its intensity; and of the degree of intensity we have no precise measure; and hence a light sleep may exhibit a change not far removed from that of wakefulness; and awaking from a light sleep may scarcely alter the rate observed during that sleep. 3d. Involuntary attempts to prevent cough in sleep retard respiration, for a limited period at a time, and thereby lessen the respirations during sleep at the moment of examination; whilst the fit of coughing in which the patient often wakes increases the respirations, so that they are then beyond the normal rate of waking.

\section{A. Waking from Sleep.}

In the examinations detailed in Diagrams Nos. 1 and 2, I usually found that this effect was to lower the rate from 5 to 8 respirations per minute. In the examination on November 14-15, the effect was precisely noted in fourteen instances, and recorded. In two instances no change occurred (14 and 21 per minute), but in the remaining twelve, the decrease with waking was as follows: 18, 12, 10, 7, 6, $6,5,4 \frac{1}{2}, 3,3,2 \frac{1}{2}$, and $1=6.5$ medium.

To this may probably be added some unknown quantity, for it is highly improbable that the whole decrease took place in one minute, judging from what $I$ have seen in health. No observation was made at a later hour than 3 a.m., for after that the patients either remained asleep during the whole examination, or were fully awake at the examination; but there is reason to believe that the decrease referred to is greatest in the early part of the night. It is also certain that, when the respirations are not much beyond 20 per minute, the decrease on awaking is small, and that so great 
a decrease as 18 or even 10 respirations cannot occur with that rate of respiration ; but it does not appear that the decrease is altogether proportioned to the frequency of the act, except when conjoined with depth of sleep.

\section{B. Hourly changes from Sleep to Wakefulness.}

There was much variation in the returns, and especially in the first night, when the novelty of the examination disturbed the rest of the patients; but the rule is that the respirations are more frequent in an examination during sleep than during wakefulness.

The following are instances supporting the rule :

Diagram No. 1.-June 11. 4, 7, and 8 a.m. ; 4 and 11 p.m. June 12. 4 and 8 a.m.; 11 p.m. June 13. 1, 4, 6, 7, and 8 a.m.; 4, 11, and 12 p.m. June 14. 1, 5, 6, 7, 8, and 11 a.m. ; 2 and 11 p.m. June 15. 2, 4, and 7 a.m.

Diagram No. 2.-June 11. 1, 2, and 7 a.m. June 12. 1, 2, 3, 4, and 8 a.m. June 13. 6 a.m.; 11 and 12 p.m. June 14. 7 a.m.; 11 and 12 p.m. June 15. 4, 5, and 7 a.m. June 16. 5, 6, and 8 a.m.

The amount of the increase observed at the hour of sleep, above the respiration at the hour of waking, varied very greatly. In Case 45, it was once 15; Case 43, 17 (two succeeding hours); Case 15, 17 $\frac{1}{2}$ and 15 ; but when observed twice in the same case, it was never precisely the same. It was often very great in Cases Nos. 35, 73, and 80 (Diagrams 1 and 2). In Case 80, the increase during the first hour of sleep was very striking, and in the six days was as follows : $8,15,12,10,9 \frac{1}{2}$, and $12=11$ respirations medium. In Case 35 (woman), it was also 11, 9, and $12=10 \cdot 6$ medium.

The highest rate of respiration commonly occurred in the early part of the night, and the respirations were fewer as the morning advanced, but in some instances, after the subsidence at 2, 3, or 4 a.m., there was another marked increase at 5, 6, or 7 a.m., corresponding with what is 
known popularly as second sleep. So long as sleep in any degree remained, the respirations were more frequent than in the hour of permanent waking; and in the instance of second sleep, in Case 34, the fall of respiration from the last hour of sleep to the first of permanent waking was, in the six days, so great as $12,11,18,12,12=13$ respirations medium.

\section{Influence of Food over hourly rate of Respiration.}

Diagram No. 4 is available only for the women, since the meal hours for the men varied during the first two days. The meal hours for the women were $8 \frac{1}{2}$ a.m., $12 \frac{1}{2}$, $5 \frac{1}{2}$, and $8 \frac{1}{2}$ p.m. The following is a general description. The rate increased greatly directly after breakfast, and continued high (with or without a little further increase), until soon before dinner. Again, it suddenly increased directly after dinner, and as suddenly fell at 2 or 3 p.m., but increased again before the tea, and still further after that meal; and then finally sank rapidly, until sleep occurred. Thus it rose directly after each meal, and fell immediately before a meal, but in such a manner that the respiration before the dinner was higher than before the breakfast, and that before the tea higher than that before either of the preceding meals. The march may thus be represented by a series of platforms one higher than another, each one having ascending and descending steps. The lowest platform is before breakfast, and is gained by a descent from sleep; the second, after breakfast; the third, after dinner; the fourth, after tea, when the descent is great; and, finally, the fifth, during the sleep of the night.

The average increase from the meals was-breakfast, $1 \frac{1}{2}$; dinner, 3 ; and tea, $2 \frac{1}{4}$ respirations. The supper meal but rarely caused any increase. One case (35) had the greatest increase from dinner and tea ( 4 respirations), whilst it had no increase after breakfast; and the variations as to the 
relative influence of meals in the same day was very great.

It is difficult to estimate the average duration of this influence, since the rate progresses throughout the day, but it is probable that it was the greatest after the breakfast, whilst the amount of increase was the greatest after dinner and tea.

Thus the following facts are established:

1. There was increased rate of respiration from food, and it occurred in the first hour, and passed away more or less in the second hour. The greatest want of uniformity was evident after supper, and the least after dinner and tea.

2. The extent varied greatly in each case, but was the greatest after dinner, tea, breakfast, and supper, in their order. The extremes held the same order, and were 1 and 10,1 and $6, \frac{1}{2}$ and 5 , and 1 and $5 \frac{1}{2}$ respectively.

3. The peculiarity of each case in reference to the effect of certain meals over others was remarkable. Thus, in 73, there was no effect from any dinner, and only once from the tea, whilst the effect of both breakfast and supper was considerable. On the contrary, Cases 33 and 35 had a largely increased rate from dinner and tea, and but a very small one from breakfast.

4. The sexual differences were chiefly-

A. Women had more uniformity, rapidity, and less duration of effect. The increase during the first hour was greater after the dinner and tea meals than after breakfast and supper, whilst a decrease in the first, and often in the second hour also, was common after supper.

B. Men had a larger increase after the breakfast, with a much more frequent decrease in the first, and increase in the second hour after dinner. 
Effect of Early Rising upon the hourly rate of Respiration.

As sleep increases the respirations, and waking lessens them, so early rising lessens those of the night. The effect was varied in the different cases. Nos. 35,88 , and 80 obeyed the rule, whilst scarcely any difference was experienced by No. 34; and Nos. 33 and 80 had an increase during the first, and a decrease during the second hour. This is remarkable, since the effect upon the circulation was to increase it in all cases.

Since the breakfast hour was not changed with the early rising, the respirations were not only decreased, but kept low, and the minimum was attained somewhat earlier. The effect upon the later respirations of the day was to increase them between breakfast and dinner, in Cases 73, 33, and 88, and even until the tea hour, in Case 73 ; whilst in four cases, Nos. 33, 34, 80, and 88, they were lessened in the after half of the day. The effect upon the respirations of the whole day was too varied to be defined.

Cases Nos. 73 and 33 were unduly influenced by exercise and excitement, 35 by food and sleep, whilst 88 and 34 were less influenced by either agent.

Effect of hourly variations of Temperature upon Respiration.

I have elsewhere proved that the general daily or weekly effect of increasing temperature is to lessen the rate of respiration.

The patients lived in two temperatures, the external and the internal ; and thence the influence of one might modify that of the other. I have no records of the hourly changes in the external temperature, but it had probably little or no effect in these investigations, for the respirations were the most frequent at night, when the temperature was low, or at the lowest, and the least frequent near to midday, when, in June, the temperature must have been considerable. There is great correspondence between the lines of internal 
temperature and respiration (Diagram No. 4); but the subject is one of difficulty, since the hourly variations of temperature were but small, and the changes in respiration have been accounted for when discussing the influence of food and sleep.

The highest temperature in the wards was in the evening, and even so late as midnight. Thence it declined until 5 or 6 a.m., and remained very low until 10 or 11 a.m., and then it gradually increased until the maximum was attained at night. Thus the lines of respiration and temperature so far correspond that both are highest in the night and lowest in the morning; and thence both increase through the day. This is a sufficient correspondence to lead us at least to seek for a law of dependence, but there are three circumstances which militate against this conclusion: 1st. The hours of maximum and minimum temperature are not those of respiration. 2d. It is but very rarely that any increase in temperature is accompanied by an increase of respiration. 3d. Sudden and great changes in respiration are not accompanied by similar changes in temperature.

\section{Effect of Sunlight.}

This, although powerful upon pulsation, is null upon respiration. The effect is neither a direct nor an inverse one, since the respirations are the most frequent when there is no sunlight, and are not the most frequent when the sunlight is the most powerful. The respirations are the fewest at 8 a.m., when, in June, the sunlight is considerable; and they decline up to that hour whilst the sunlight is increasing. They also attain to a very high point in the evening, when the sunlight has declined; and the greatest and most sudden rise occurs at 1.1 p.m., when there is no sunlight. 
Ratio of the Respiration to the Pulse in Phthisis.

This varies with nearly every hour of the day and night, but is the highest in the night, when the pulsation is the lowest and the respiration the highest.

The following table exhibits the hourly ratios in all the cases combined :

TABLE No. VII.

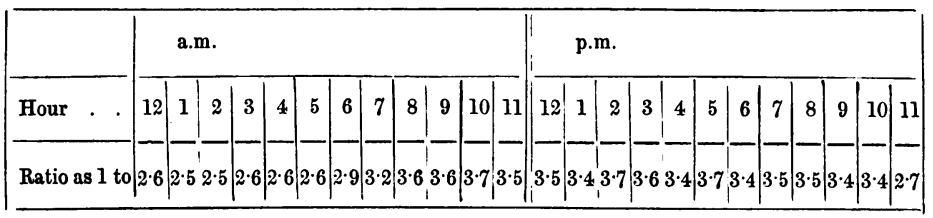

Thus the maximum was at 1 and 2 a.m., but practically it may be said to extend from 11 p.m. to 5 a.m., or the period of lowest pulsation and highest respiration. At 6 a.m., when sleep is departing, it was lowered, and continued so until 10 a.m., when it was only two thirds of the maximum. It is then at the minimum, as it is also at the hours 2 and 5 p.m. Immediately after each meal the ratio is lessened, from increased pulsation, but in an hour or two it is again increased, so that the ratio is lessened directly after each meal, and increased before each meal; but after the tea hour it does not fall; and at 11 p.m. it suddenly gains the maximum. The effect of sleep in the night is much greater than that of food, since it always raises the ratio to the maximum, by increasing respiration and lowering pulsation. The average ratio was not so low as 1 to 4, nor so high as 1 to 2 ; but the following table shows that one of the cases had a ratio as high as 1 to 4 , and never lower than 1 to $2 \cdot 3$. The lowest ratio observed was 1 to $5 \cdot 8$, but in the early stages of the disease it is often lower than that. Thus, in a soldier, six feet two 
inches in height, a patient of mine, now at the hospital, it was, on November 14th, only 1 to 8 in the lying posture.

TABLE No. VIII.

Exhibiting the Average Ratios in each of the cases, and the average in each sex.

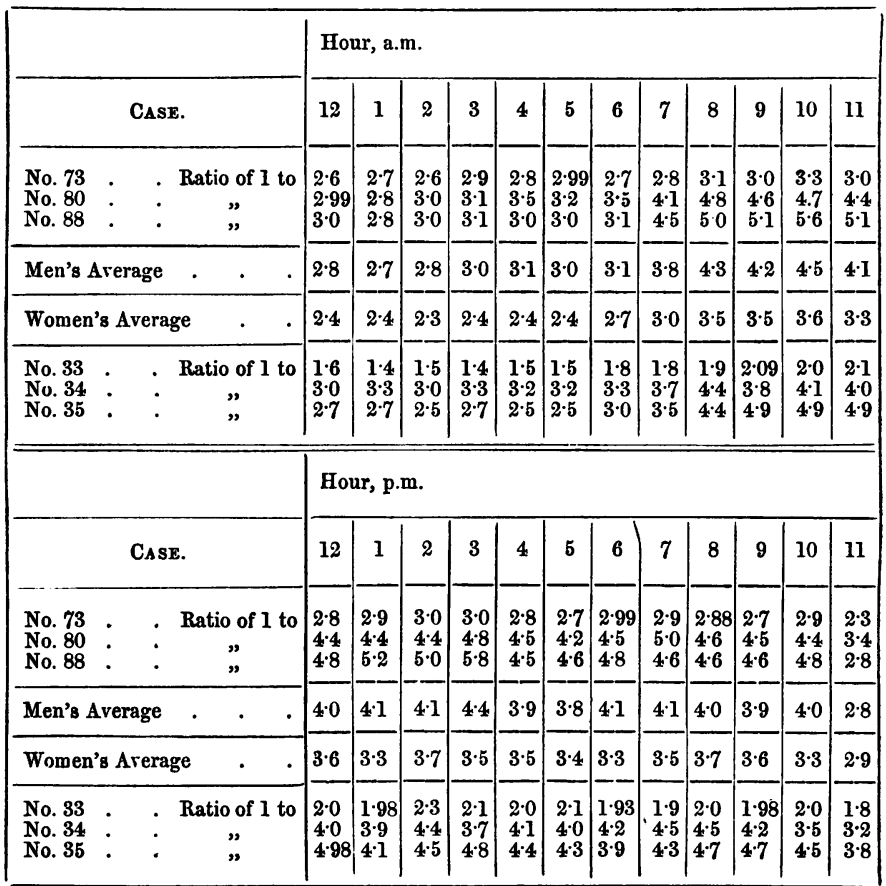

Thus whilst the ratios in each case varied much, and were much higher at every hour in the women than in the men, they support the statements made from the total average. No. 34 had, however, a less proportionate night increase than any other, so that its maximum was 1 to $3 \cdot 0$, and its minimum 1 to $4: 5$. The maximum hour in all was from 11 p.m. to 3 a.m., and once extended even to 4 and 5 a.m., whilst the minimum was from 10 a:m. to 8 p.m. 
The maximum varied from as 1 to $1 \cdot 4$, to as 1 to $3 \cdot 0$ In some instances. the ratio somewhat increased directly after the meal.

\section{Summary.}

The following is a summary of the foregoing communication :

1. The rate of both pulsation and respiration varies with each hour of the day and night; that of pulsation on the average of the day to 26, and of respiration to 8 per minute.

The rate of pulsation is the lowest in the "night" ( 1 to 5 a.m.), and the highest in the "day" (9 a.m. to 9 p.m.), and rises greatly in the " morning" (5 a.m. to 10 a.m.), and sinks greatly in the "evening" (9 p.m. to 1 a.m.) The rate of respiration is the greatest in the night, and the least at about 8 a.m.; after which it increases until about 6 or 7 p.m., when it declines to the hour of sleep; so that it is the least before breakfast, then before dinner, then before tea, and the highest when the patient is asleep at night.

2. The average variation of pulsation is greatest in the " morning" (27), then in the "evening" (19), " day" (7), and "night" (10).

3. The night minimum of pulsation (65) was most frequently at 5 a.m., and the day minimum (84) was 20 pulsations above it. The day maxima were (breakfast) 95, (dinner) 97, (tea) 96, and (supper) 92.

4. In women the pulsation was lower and more uniform, and with a greater night subsidence and less morning elevation. The respiration was quicker and more uniform, and the sudden increase at night was not so great, because the respiration did not fall so low after tea.

5. The day increase of pulsation is chiefly due to food and sunlight, but of respiration to food alone; whilst the decreased night pulsation is due to darkness and sleep, and the increase of respiration to sleep only. The effect of sound sleep was to lower pulsation 8 to 10 , and increase 
respiration about 10 per minute; whilst light sleep may be estimated from one half of these numbers to nothing.

Thus during sound sleep the pulse falls greatly for two or three hours, and the respirations are at their highest; but as the morning advances; and whilst the patient is yet asleep, the pulse rises and the respiration falls. When second sleep occurs, at 4, 5, 6, or 7 a.m., the respirations are again increased, and then the fall on final waking averaged 13 respirations per minute. The pulse is not commonly depressed by the second sleep. The deeper the sleep, the more frequent the respirations; but the pulsation is not always then the lowest. The pulse falls and the respirations rise on sleeping, and both the contrary on waking out of sleep.

6. Food increases both pulsation and respiration, on the average, as follows: Breakfast 8 to 10 and $2 \frac{1}{4}$; dinner 11 and 3 ; tea 7 and $1 \frac{1}{2}$; and supper $2 \frac{1}{2}$ pulsations. There is great variation in both functions.

The maximum effect was earlier in respiration than pulsation, and in the former was commonly within one hour, except after supper, and endured longest after breakfast; whilst in the latter (pulsation) it was chiefly the second hour, and was the second, first, third, and fourth hour in their order of frequency. In women the effect on respiration was more uniform, rapid, and evanescent.

7. Fasting (for a short time) caused more pulsation, and in one half of the cases more frequent respiration also.

8. Early rising increased pulsation before breakfast and before dinner, but lessened it in the after part of the day, and in the day taken as a whole, and it produced an earlier and a deeper sleep. It lessened greatly the respirations before breakfast, and sometimes increased those of the afternoon.

9. Hourly changes of temperature correspond more with respiration than pulsation, but probably are not causative in either function.

10. Moderate exercise has no appreciable effect on either function if the lying posture be maintained afterwards for a few minutes. 
11. The effect of sunlight, in June, is to increase pulsation on an average of 6 to 10 per minute; but it has no influence on respiration.

12. There is a disposition in both functions to rise at the wonted hours of rising, as seen in the short fast.

13. On several occasions the rate-of both functions suddenly halved or doubled itself, as in Case 35, 4th day, Case 33, 2d day, Case 34, 1st day; all at the same hour, viz., 2 p.m.

14. Upon the whole the rate of the functions was the least disturbed in the night and the working day, and then the effect of the respective influences was the most perfect, whilst the characteristic of the morning and evening was " change."

15. The ratio of respiration to pulsation was on the average not so low as 1 to 4 nor so high as 1 to $2 \cdot 3$; but it was much higher in women than in men; and in the case having the highest ratio it varied from as 1 to $1 \cdot 4$ to as 1 to $2 \cdot 3$, and in that having the lowest it varied from as 1 to $2 \cdot 8$ to as 1 to $5 \cdot 8$. It was the highest at night (from about 11 p.m. to 5 a.m.) and the lowest from 10 a.m. to 5 p.m. It was lowered directly after a meal and raised before a meal, and was the highest with sleep in the night.

The following are the chief variations from a state of health :

1. The rate of both functions is greater.

2. The night pulsation is scarcely beyond that of health; but the day pulsation is upwards of 20 pulsations greater, and is more than double of the healthy increase. Thus the chief variation in pulsation refers to the day.

3. The ranges of pulsation at each of the four periods of the day are much greater, but especially so in the "evening" and " morning."

4. The respirations in the night and also in the day are directly opposed; but both in health and disease they are increased generally after meals.

5. The effect of food upon pulsation is not widely different. It is a little less at breakfast and a little more at tea and supper, whilst it is equal at dinner. The effect upon respi- 
ration was greater at dinner, less at breakfast, and equal at tea.

6. The ratio of the two functions is much higher, and is opposed in its hourly changes, since it is highest in the night and lowest in the day. also.

7. The effect of sleep is greater, and probably of sunlight

But in making the above statements we must not forget that the investigation for disease was made in June, with powerful sunlight, and that for health in November, with but little sunlight power.

\section{Deductions.}

The chief deductions from this investigation may be made with more truthfulness when I shall have ascertained the quantity of air inspired. Only a few will, therefore, be now presented.

1. As profuse perspirations occur in phthisis during sleep, and as, during both day and night sleep, the pulse is lowered, and that to a very great extent in the night, it is probable that the former much depends upon the latter. With this impression I have administered food with wine or cold tea in the night with great advantage in preventing the perspirations. As milk scarcely increases pulsation, it has not been of great service for this purpose. Further, as day sleep is accompanied by a rate of pulsation much above that attending upon night sleep, it is probable that the exhaustion which induces the perspiration would be somewhat prevented by cultivating day sleep and curtailing the hours of night sleep. As the pulse falls in the evening and rises in the early morning, under all circumstances, $I$ believe that to retire to rest at 8 or 9 p.m. and to rise at 6 a.m. would save the system in phthisis; but with the early rising it is essential that there should be an early breakfast.

2. As the influence of sunlight is so powerful at Midsummer in increasing the rate of pulsation so much above 
the condition of the pulse in darkness, it may be inferred that too free exposure to it would exhaust the system. On the other hand, the deficiency of sunlight in winter, and more particularly in close, dark streets, alleys, and rooms, is likely to maintain the state of low vitality which is so essential a part of the disease. Free exposure to sunlight in winter and restricted exposure in summer seem therefore to be indicated.

3. The extremely low rate of pulsation obtained and maintained for so many hours of the night, with, at the same time, the greatly increased rate of respiration during that period, indicate the necessity for the administration of nutritive food once or more frequently through the night. I believe this to be very important in preventing the exhaustion attending upon low pulsation, and also as permitting the meals taken during the day to be moderate in quantity, and thereby to prevent much of the unnatural and exhausting day elevation of the pulse. To this end it is probable that the administration of carbonaceous matter, as cod-liver oil, the last thing at night, immediately before the great increase in the rate of respiration from sleep, would be beneficial. But the night increase in respiration is not found in every case.

4. The ratio which the respiration bears to pulsation must be important in phthisis both in prognosis and in treatment. This, other conditions being similar, is influenced differently in different cases. Thus in one the ratio shall be lessened by increase of pulsation from excitement during the day, whilst in others excitement shall have but little influence, and in them the ratio shall be lessened by very profound sleep, which lessens pulsation and increases respiration. In order, therefore, to valuable deduction, it is needful to compare the day with the night, and not to trust to absolute numbers. However injurious rapidity of both functions may be, it cannot be but that rapid pulsation with slow respiration must be more so. Hence, when the ratio is so low as 1 to 7 or 1 to 8 , it is open to inquiry if such a condition will not necessarily lead to impoverishment of the 
system. Hence the aim would be to increase respiration disproportionately to pulsation. This is probably effected by remedies which give tone to the system; but it is now proved to be so by food, and especially by sleep, and hence the due cultivation of the horizontal posture with frequent food and day sleep are indicated. Early retiring to rest has also the same effect. I have noticed the low ratio referred to chiefly in phthisical persons of unusual stature, as in some of the regiments of Life Guards, and in the early stage. Women appear to obtain a sounder sleep than men, for in them the pulse is more quickly reduced and reduced to a lower point at night, whilst, at the same time, the rate of respiration is greater than in men. The cause of the night increase in the rate of respiration in phthisis cannot be ascertained until the quantity of air inspired is determined. 
No 4.

Average hourly Respirations during 6 days \& nights in 6 Phthisical persons,

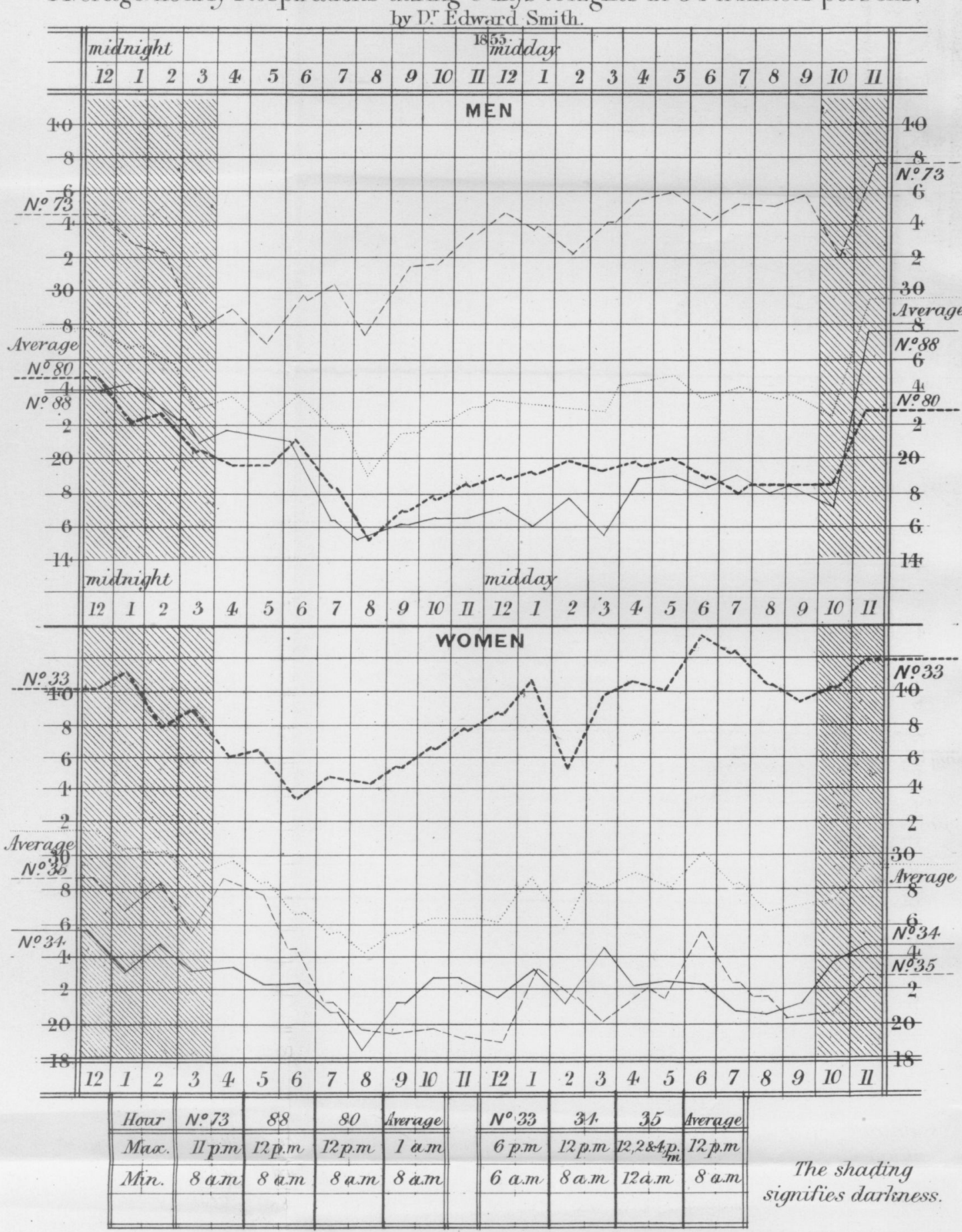

Average hourly Temperature of the Wards.

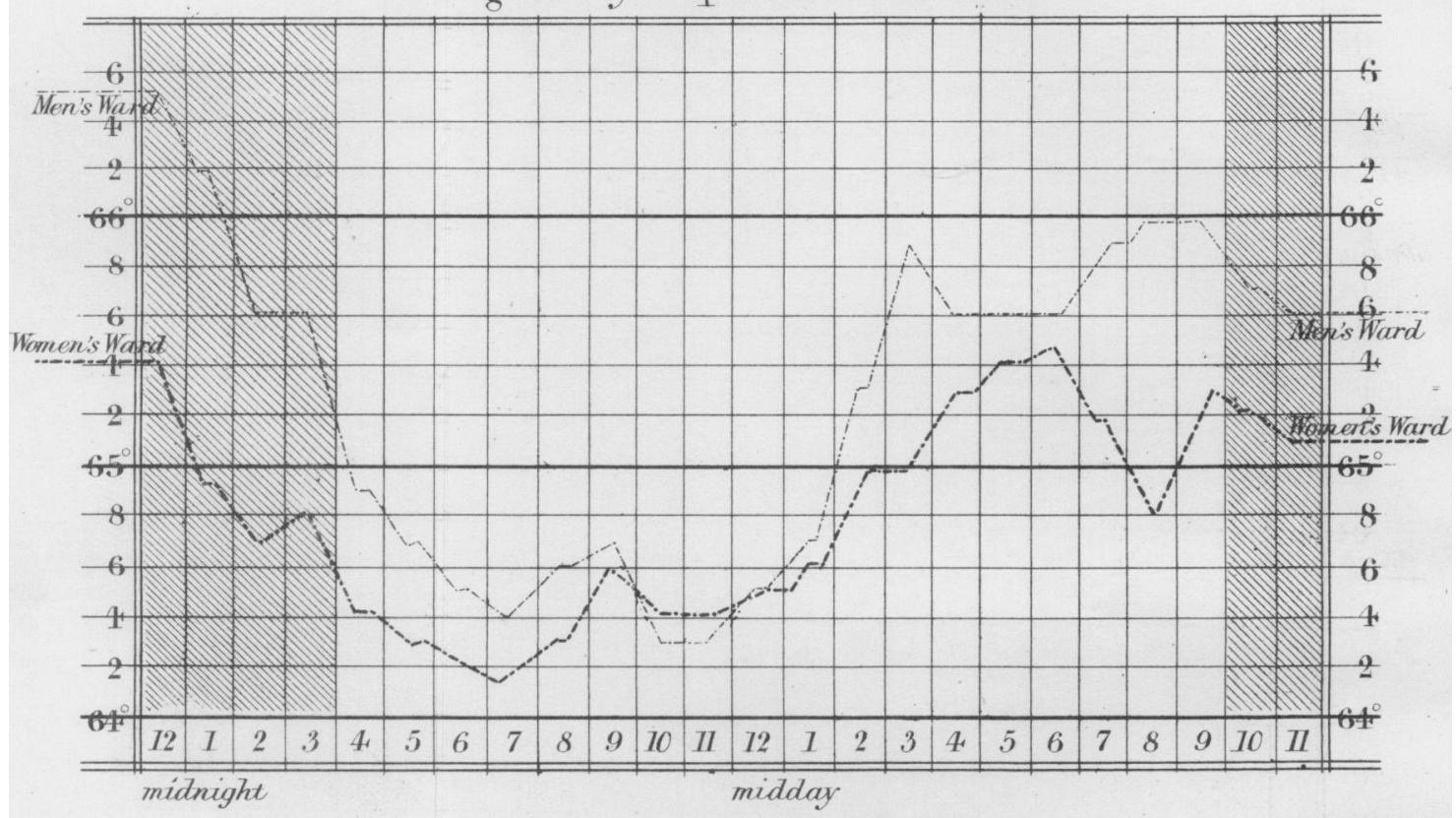




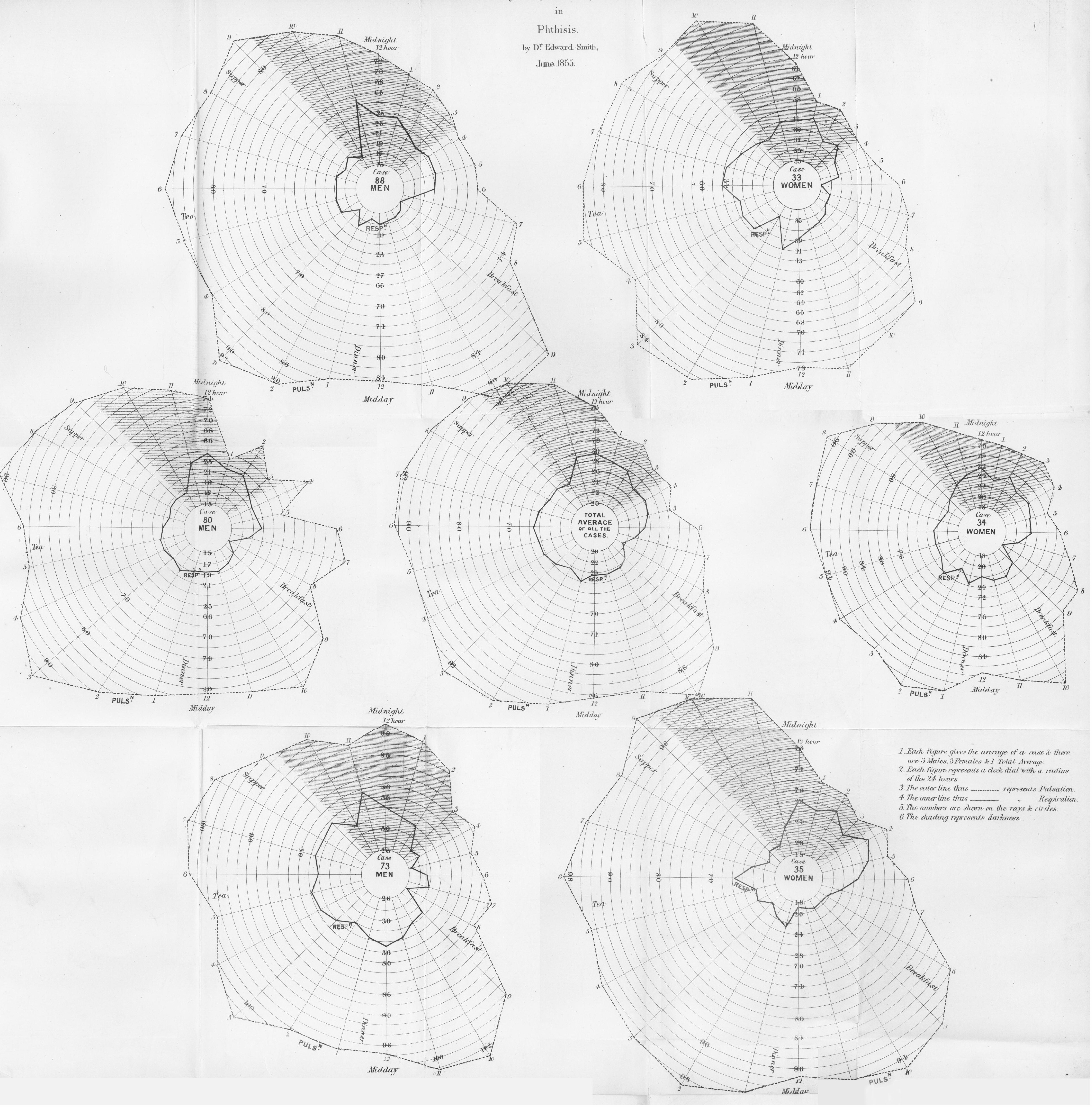




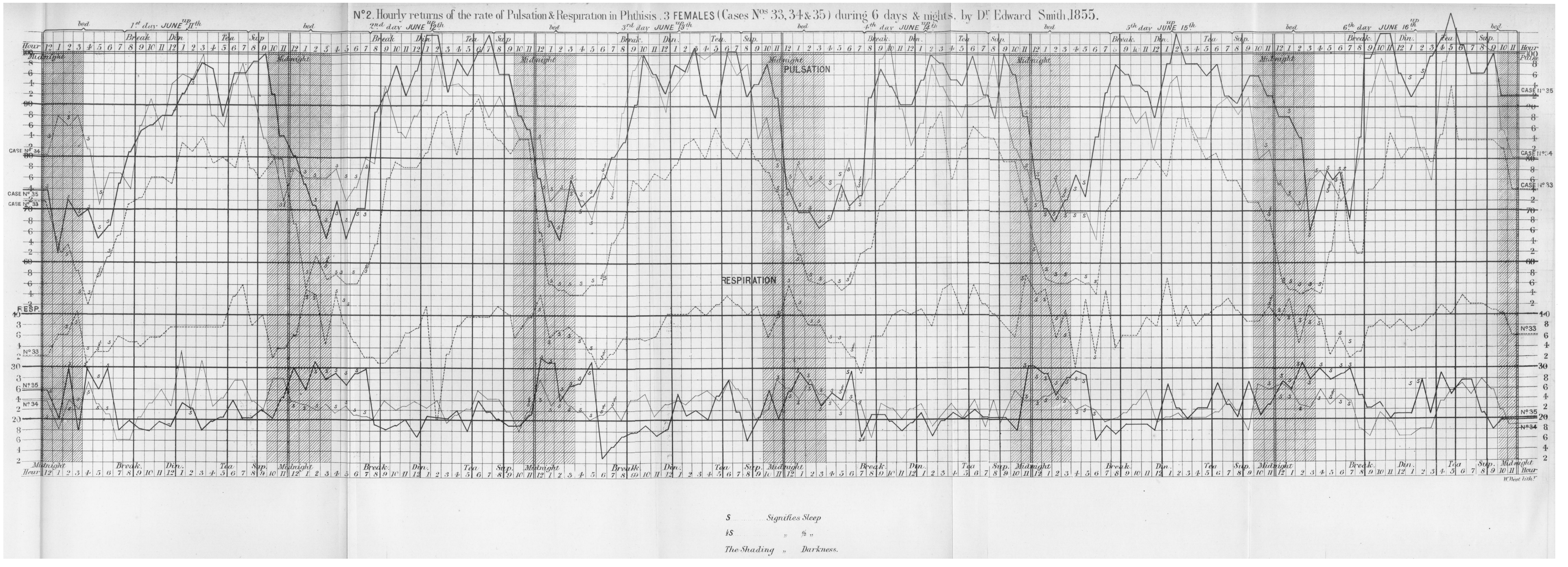





\section{EXPLANATION OF THE DIAGRAMS ACCOMPANYING THE PAPER ON THE HOURLY RATE OF PULSATION AND RESPIRATION IN PHTHISIS.}

Nos. 1 and 2 are constructed on one principle, and refer, the former to three men, and the latter to three women. They represent the rate of both functions, in the lying posture, at each of 144 consecutive hours, or six days and nights ; the upper series of lines in each diagram being devoted to the pulsations, and the lower to the respiration in each case. The series of vertical double lines indicate the hour of midnight, whilst the intervening spaces are each divided into twenty-four columns, each indicating one of the twenty-four hours of the day. The thinner vertical lines show the hours of meals in each case on every day; and the horizontal lines, with the scale on either hand of the diagram, indicate the rate of both functions at every hour of the inquiry. The shaded parts include the hours of darkness in May and June, and the white parts the period of sunlight. The letter $S$ shows that the patient was asleep at that hour, whilst $\frac{1}{2} \mathrm{~S}$ signifies half-sleep, or a state which did not appear to the observer to be true sleep.

No. 3 exhibits the same facts, on an average derived from 144 hours. The six outer figures are devoted to the six cases, and the central figure to the average of the whole combined. Each figure resembles a clockdial with twenty-four radii or hours, the outer boundary line showing the number of pulsations, and the inner line the number of respirations at each hour. Each radius or hour is numbered, and the scale of pulsation and respiration is attached to the concentric circles. The shading indicates the hours of darkness, and the white part the hours of sunlight. The period of meals is also given on each figure.

No. 4 shows, in the upper part, the average hourly respirations, in the lying posture, in each of the cases, with the averages of all the cases of each sex, and the hours of darkness and of light; and in the lower part the average hourly temperature of each of the two wards occupied by the cases under inquiry. 